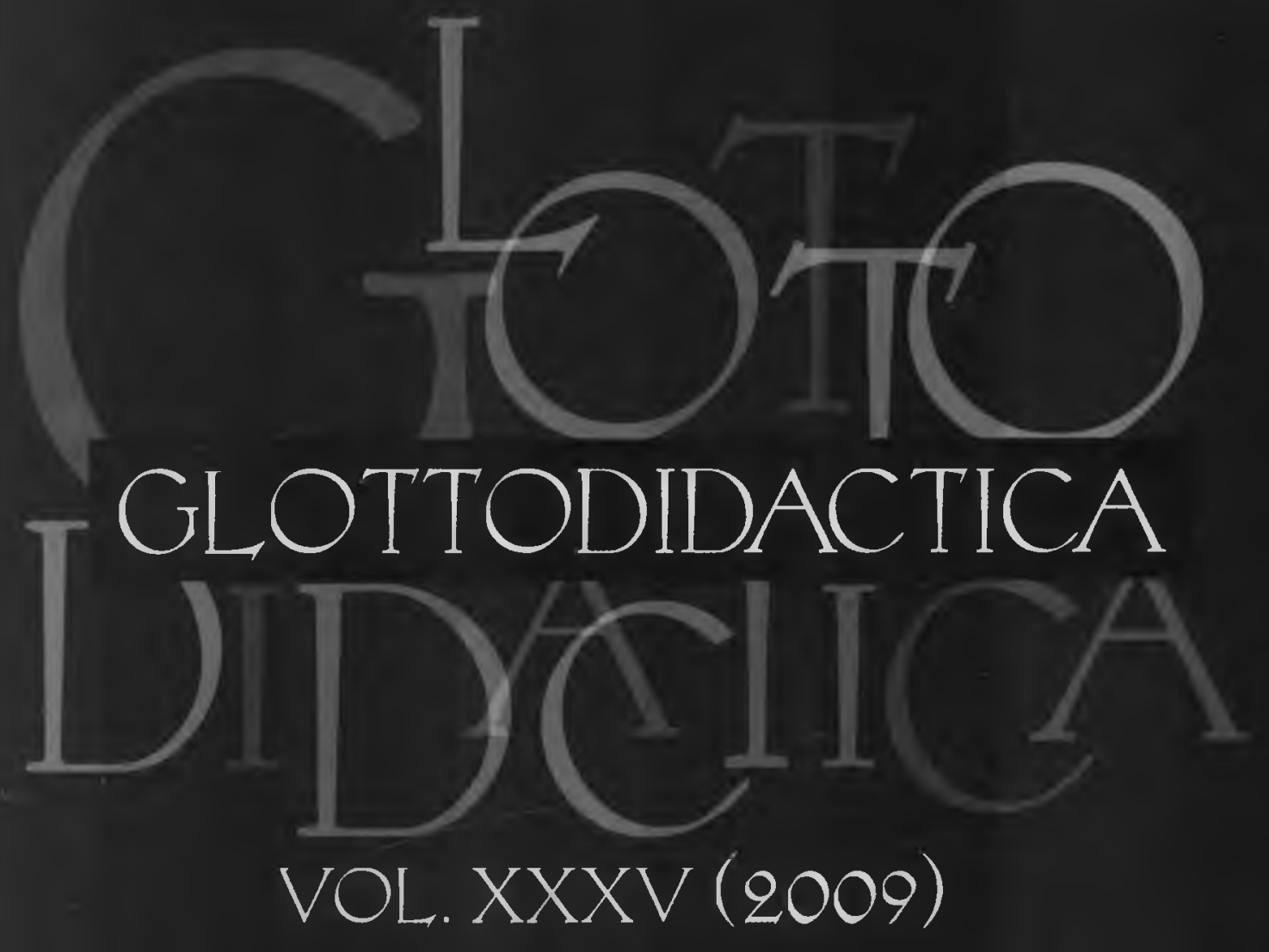

WYDAWNICTWO NAUKOWE UAM 



$$
\operatorname{cod} .42582 \wedge \frac{11}{1309}
$$

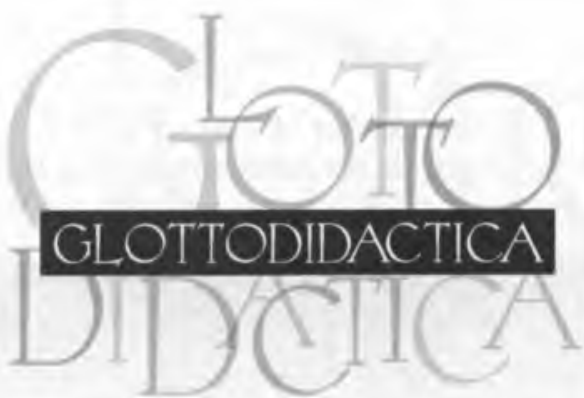




\section{GLOTTODIDACTICA \\ An International Journal of Applied Linguistics \\ Founding Editor: Prof. Dr. Ludwik Zabrocki}

Ausgabe / Issue 35

Herausgeber / Publisher

Institut für Angewandte Linguistik der Adam Mickiewicz Universität Poznań

V.i.S.d.P. Responsible person in accordance with Polish press law

Prof. Dr. Waldemar Pfeiffer (Honorary Editorship)

Redaktion (Leitung) / Editorial Head

Prof. Dr. Barbara Skowronek

Redaktionelle Mitarbeit / Editorial support

Dr. Luiza Ciepielewska-Kaczmarek, Dr. Monika Kowalonek-Janczarek

Redaktionsbeirat Editorial / Advisory Board

Prof. Dr. Jerzy Bańczerowski, Prof. Dr. Anna Cieślicka, Prof. Dr. Józef Darski,

Prof. Dr. Jacek Fisiak, Prof. Dr. Antoni Markunas, Prof. Dr. Kazimiera Myczko,

Prof. Dr. Stanisław Puppel, Prof. Dr. Christoph Schatte, Prof. Dr. Teresa Tomaszkiewicz, Prof. Dr. Weronika Wilczyńska, Prof. Dr. Stephan Wolting, Prof. Dr. Władysław Woźniewicz

Wissenschaftlicher Beirat / Academic Advisory Board

Prof. Dr. Rupprecht S. Baur (Duisburg/Essen), Prof. Dr. Dmitrij Dobrovolskij (Moskau/Wien), Prof. Dr. Franciszek Grucza (Warszawa), Prof. Dr. Wolfgang Herrlitz (Utrecht),

Prof. Dr. Hanna Komorowska (Warszawa), Prof. Dr. Bogdan Kovtyk (Halle),

Prof. Dr. Frank Königs (Marburg/L.), Prof. Dr. Roman Lewicki (Wroclaw),

Prof. Dr. Clare Mar-Molinero (Southhempton), Prof. Dr. Paweł Mecner (Szczecin),

Prof. Dr. Bernd Müller-Jacquier (Bayreuth), Prof. Dr. David S. Singleton (Dublin), Prof. Dr. Marian Szczodrowski (Gdańsk), Prof. Dr. Iwar Werlen (Bern), Prof. Dr. Jerzy Żmudzki (Lublin) 
UNIWERSYTET IM. ADAMA MICKIEWICZA W POZNANIU

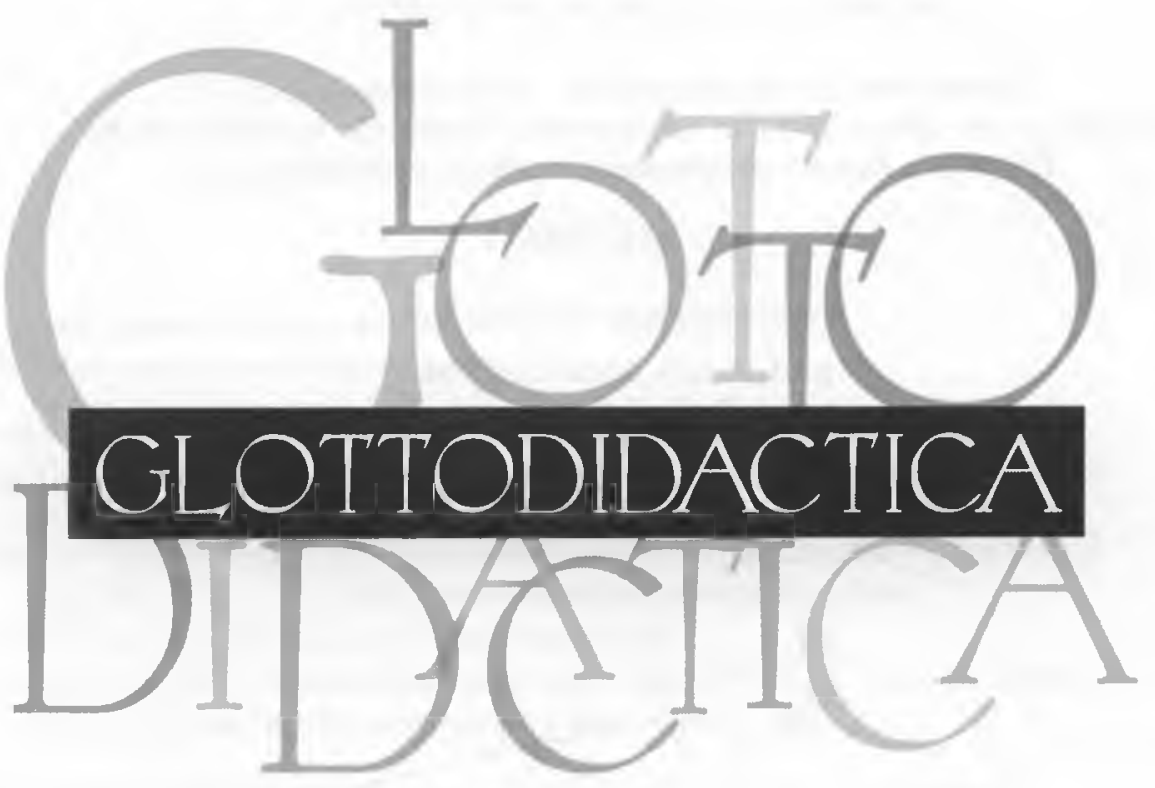

VOLUME XXXV

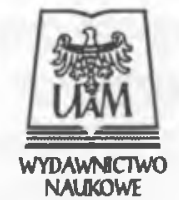

POZNAŃ 2009 


$$
\begin{gathered}
\text { Adres redakcji } \\
\text { Instytut Lingwistyki Stosowanej UAM } \\
\text { ul. } 28 \text { Czerwca } 1956 \text { r. nr } 198 \\
\text { 61-485 Poznań, Poland } \\
\text { tel. }+48618292925 \text {, tel./ fax }+48618292926
\end{gathered}
$$

Editor: Prof. Barbara Skowronek barbaras@amu.edu.pl Assistants to the Editor: Dr Luiza Ciepielewska-Kaczmarek luizac@poczta.fm

Dr Monika Kowalonek-Janczarek monika.kowalonek@wp.pl

$$
\begin{gathered}
\text { Weryfikacja językowa tekstów } \\
\text { prof. dr hab. Anna Cieślicka } \\
\text { dr Britta Stöckmann }
\end{gathered}
$$

Publikacja dofinansowana przez

Instytut Lingwistyki Stosowanej UAM

\section{POZNANI}

(C) Wydawnictwo Naukowe UAM, Poznań 2009

Wydano na podstawie maszynopisu gwarantowan go

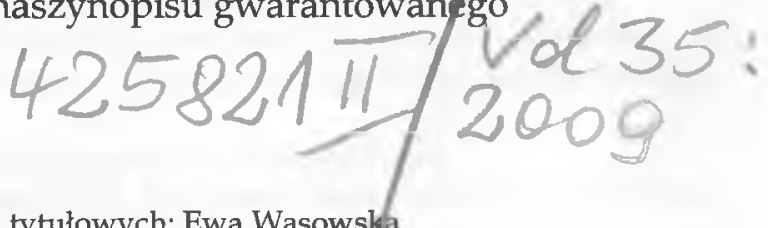

Projekt okladki i stron tytułowych: Ewa Wąsowska

Redaktor prowadzący: Anna Rąbalska

Formatowanie: Eugeniusz Strykowski

\section{ISBN 978-83-232-2083-1 ISSN 0072-4769}

WYDAWNICTWO NAUKOWE UNIWERSYTETU IM. ADAMA MICKIEWICZA

61-734 Poznań, ul. F. Nowowiejskiego 55, tel. 06182939 85, fax 0618293980 e-mail: press@amu.edu.pl www.press.amu.edu.pl

Ark. wyd. 17,00 . Ark. druk. 15,50

ZAKŁAD GRAFICZNY UAM, POZNAŃ, UL. H. WIENIAWSKIEGO 1

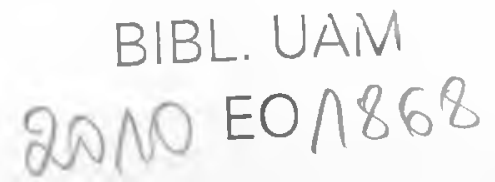




\section{CONTENTS}

\section{ARTICLES}

Basic and Referential Disciplines

Grundlagen- und Referenzwissenschaften

MARIAN SZCZODROWSKI, Wesen, Arten und Wirkungen der fremdsprachlichen Steuerung ...

GRAŻYNA LEWICKA, Zu einigen Problemen des Verstehens in der konstruktivistisch orientier-

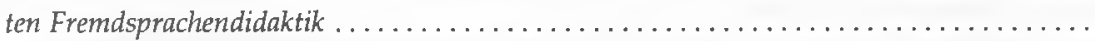

REINHOLD UTRI, Bilinguale Erziehung - neue Herausforderungen und Lösungen aus linguisti-

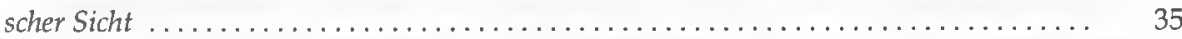

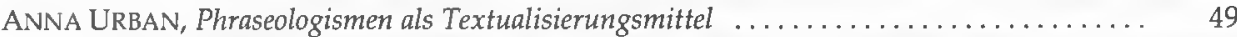

NATASCHA JOURDY, НарратиВная структура художественного текста: свободнокосвенный дискурс как категория лингвистики и нарратологии ................

\section{Methodology in Glottodidactics}

Fremdspracheninethodik

ZOFIA CHLOPEK, The relationship between language learning experience, motivation and some

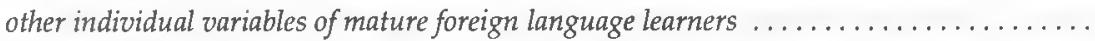

MALGORZATA CZARNECKA, Die Entwicklung interner grammatischer Regeln im gesteuerten

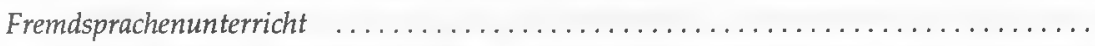

BRIGITA KOSEVSKI PULJIĆ, Der Enwerb der Schreibkompetenz im Deutsch als Fremdsprache -

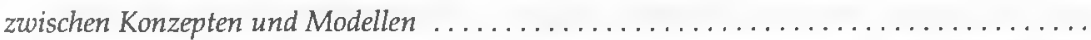

AGNIESZKA MAC, Einige Überlegungen zur Wortbildungslehre im fremdsprachlichen

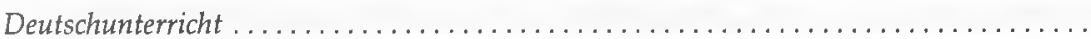

SYlWIA ADAMCZAK-KRYSZTOFOWICZ, ANTJE STORK, Podcasts im glottodidaktischen Medienverbund. Versuch einer vergleichenden Analyse ......................

MARIUSZ WALORCZYK, Optimierung der ZD-Prüfungsvorbereitung in Bezug auf die Aufgabenformen

\section{Language Policy and Intercultural Studies}

Sprachenpolitik und Interkulturelle Studien

MONIKa KOWALONEK-JANCZAREK, Die Nachbarsprache Polnisch aus einer sprachpolitischen und soziolinguistischen Perspektive im Kontext der Mehrsprachigkeit in Europa .........

NINO LOLADZE, Interkulturelles Lernen als Schlüsselqualifikation im Zeitalter der Globalisierung im Kontext des Deutschunterrichts in Georgien 


\section{RESEARCH REPORTS}

DANUTA WIŚNIEWSKA, EFL Teachers' Perception and Practice of Action Research .......... 163

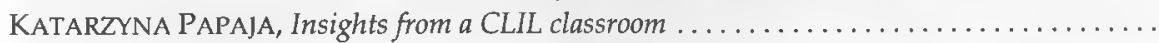

MAGDAlENA KOPER, MAGDALENA DUdZIŃSKA, Europäisierung der Sprachenpolitik im Bereich des Kulturtourismus nach dem EU-Beitritt Polens am Beispiel der Stadt Gdansk ....

\section{BOOK REVIEWS}

BARBARA SKOWRONEK, Marian Szczodrowski: Fremdsprachliche Lehr-Lern-Vorgange im kodematischen Blickfeld. Wydawnictwo Uniwersytetu Gdańskiego, Gdańsk - Sopot 2009,225 S. . . . . . . . . . . . . . . . . . . . . . . . . . . . . . .

JOANNA KIC-DRGAS, Sambor Grucza: Lingwistyka języków specjalistycznych. Wydawnictwo Euro-Edukacja, Warszawa 2008, 256 S.

ANNA PIECZYNSKA-SUlIK, Hans-Jörg Schwenk: Präfigierung im Polnischen und ihre Entsprechung im Deutschen. $\mathrm{Zu}$ Theorie von und lexikographischem Umgang mit Aspekt und Aktionsart. Reihe: Języki - Kultury - Teksty - Wiedza. Band 8, Wydawnictwo EuroEdukacja, Warszawa 2007, $301 \mathrm{~S}$.

BARBARA SKOWRONEK, Magdalena Olpińska: Nauczanie dwujęzyczne w świetle badań $i$ koncepcji glottodydaktycznych. Seria: Języki - kultury - teksty - wiedza. Franciszek Grucza, Jerzy Lukszyn (Hg.). Wydawnictwo Euro-Edukacja, Warszawa 2008, 247 S. . . . . . .

REINHOLD UTRI, Marc Bielefeld: We spe@k Deutsch... aber verstehen nur Bahnhof. Unterwegs im Dschungel unserer Sprache. Wilhelm Heyne Verlag, München, 2008, 286 S.

GABRIELA GORĄCA, Sambor Grucza: Od lingwistyki tekstu do lingwistyki tekstu specjalistycznego. Seria: Języki - kultury - teksty - wiedza. Franciszek Grucza, Jerzy Lukszyn (Hg.). Wydawnictwo Euro-Edukacja, Warszawa 2007, 218 S. .............

KATARZYNA KONSEK, Jörg Roche: Handbuch Mediendidaktik. Hueber Verlag, Ismaning

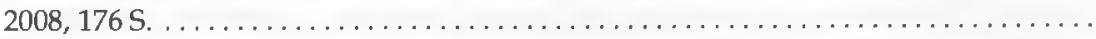

ANNA URBAN, Christiane Hümmer: Synonyme bei phraseologischen Einheiten. Eine korpusbasierte Untersuchung. Peter Lang, Frankfurt am Main 2009, 357 S. . . . . . . . .

SYLWIA ADAMCZAK-KRYSZTOFOWICZ, Kazimiera Myczko, Barbara Skowronek, Władyslaw Zabrocki (red.): Perspektywy glottodydaktyki i jezykoznawstwa. Tom jubileuszowy $z$ okazji 70. urodzin Profesora Waldemara Pfeiffera. Wydawnictwo Naukowe UAM, Poznań 2008, 499 S. . . . . . . . . . ..............................

MONIKA KOWALONEK-JANCZAREK, Boris Blahak, Clemens Piber (Hg.): Deutsch als fachbezogene Fremdsprache in Grenzregionen. Ekonóm, Bratislava 2008, 322 S. . . . . . . .

LUIZA CIEPIELEWSKA-KACZMAREK, Sambor Grucza (Hg.): W kręgu teorii i praktyki lingwistycznej. Księga jubileuszowa poświęcona Profesorowi Jerzemu Lukszynowi z okazji 70. rocznicy urodzin. Wydawnictwo Uniwersytetu Warszawskiego, Warszawa 2007, 367 S. . . . . . . . .

AGNIESZKA PAWŁOWSKA, Kultury i języki poznawać - uczyć się - nauczać / Kulturen und Sprachen verstehen - lemen - lehren. Pod redakcją / Herausgegeben von Anna Jaroszewska, Marta Torenc. Uniwersytet Warszawski, Instytut Germanistyki, Warsza-

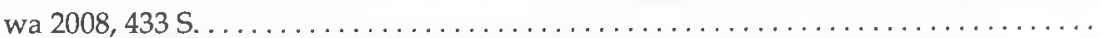

BEATA MikOŁAJCZYK, Konrad Ehlich, Dorothee Heller (Hg.): Die Wissenschaft und ihre Sprachen. 2006. Peter Lang Verlag, Bern / Berlin / Bruselles / Frankfurt a.M. / New York / Oxford / Wien. (= Linguistic Insights. Studies in Language and Communi-

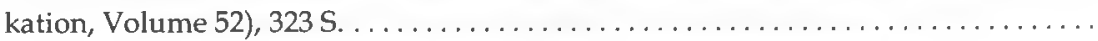




\section{REPORTS}

LUIZA CIEPIELEWSKA-KACZMAREK, Lesen und Leseverstehen in der DaF-Didaktik

MAGDAlena JUREWICZ, PAWEE RYBSZLEGER, Bericht über die Internationale wissenschaftliche Konferenz des Verbandes Polnischer Germanisten (VPG) ..........

AGNIESZKA PAWŁOWSKA, Bericht über die internationale Konferenz Reflexion als Schlüsselphänomen der gegenwärtigen Fremdsprachendidaktik

GRAŻYNA ZENDEROWSKA-KORPUS, Das linguistisch-hochschuldidaktische Kolloquium „Fachsprachenpropädeutik im Germanistikstudium" 



\title{
Optimierung der ZD-Prüfungsvorbereitung in Bezug auf die Aufgabenformen
}

\section{Optimizing Certificate German (ZD) Test Preparedness with Regard to Form of Task}

\begin{abstract}
Optimizing Certificate German (ZD) Test Preparation with Regard to Task Form Placement and progress tests are used to provide optimum instruction. Experience with test task forms at various times in various situations increase task related skills and reduce test anxiety - closed tasks exercising recognition; open tasks production. Very important are skills in comprehending test task instructions and carrying them out. As an alternative to the more stressful schoolroom work, E-tests and CD-ROM work facilitate and stimulate preparation so long as the desired goals are kept in the forefront.
\end{abstract}

Keywords: optimize, standardized, German, test, preparedness, priorities, placement, progress, tasks, instructions.

Die international anerkannte Prüfung zum Zertifikat Deutsch (ZD) ist das Ergebnis einer Kooperation zwischen dem Goethe-Institut (GI), dem Österreichischen Sprachdiplom (ÖSD), der Schweizer Erziehungsdirektorenkonferenz (EDK) und der TELC GmbH (WBT Weiterbildungs-Testsysteme $\mathrm{GmbH}$ ). Das Zertifikat Deutsch orientiert sich hauptsächlich an den Bedürfnissen von Lernenden in der Erwachsenbildung im In- und Ausland. Neuere Entwicklungen stellen das gleiche Prüfungsformat auch jugendlichen Lernenden zur Verfügung. In der Regel brauchen Lernende zwischen 350 bis 600 Unterrichtsstunden, um die notwendige Sprachbeherrschung für eine erfolgreiche Prüfung zum Zertifikat Deutsch zu erlangen. Das Zertifikat Deutsch orientiert sich an der Stufe B1 des GER (Europarat 2001) - der international anerkannten Skala des Europarates und hat weltweit einen hohen 
Bekanntheitsgrad und wird von privaten und öffentlichen Arbeitgebern als Nachweis von soliden Grundkenntnissen in der deutschen Sprache geschätzt. In Deutschland ist das ZD als Nachweis von Deutschkenntnissen zur Erlangung der deutschen Staatsangehörigkeit anerkannt.

Die Lernzielkataloge, die dem Zertifikat Deutsch zu Grunde liegen, beruhen auf dem Sprachgebrauch des Standarddeutschen in der Bundesrepublik Deutschland, in Österreich und in der Schweiz. Das ZD löst das Zertifikat Deutsch als Fremdsprache (ZDaF), das von 1971 bis 1999 vom Deutschen Volkshochschulverband und dem Goethe-Institut vergeben wurde und die ÖSD-Grundstufe 2, die es seit 1994 gab, ab. Heutzutage nimmt das ZD einen neuen Namen „ZD-B1“ an.

Das Zertifikat bescheinigt gute Kommunikationskompetenz in Situationen des täglichen Lebens. Es gilt daher für viele Institutionen als entsprechender Nachweis. In einzelnen Fällen kann es auch als Voraussetzung für die Teilnahme an weiterführenden Sprachkursen erwartet werden. Das ZD-B1 ist seit dem 01.01.2005 die abschließende Sprachprüfung in Integrationskursen nach dem Zuwanderungsgesetz. Diese Prüfung für Deutschlernende $a b$ 16 Jahre stellt den Nachweis der Fähigkeit zur selbstständigen Sprachverwendung in Alltags- und Berufssituationen dar. Für das Bestehen des Zertifikats Deutsch sollten Kandidatinnen und Kandidaten fähig sein, sich in vertrauten Situationen sprachlich problemlos zurechtzufinden. Mit dem Bestehen der Prüfung ZD-B1 weisen die Kandidaten nach, dass sie über solide Grundkenntnisse in der deutschen Umgangssprache verfügen, die es ihnen ermöglichen, sich in allen wichtigen Alltagssituationen sprachlich zurecht zu finden. Sie zeigen mit dem ZD-B1, dass sie die wesentlichen grammatikalischen Strukturen beherrschen und im Stande sind, ein Gespräch über Situationen des täglichen Lebens zu verstehen und sich daran zu beteiligen. Ebenso sind sie fähig, einfache Sachverhalte mündlich und schriftlich darzustellen und Texte $\mathrm{zu}$ Alltagsthemen zu verstehen. Lernende können mit dem ZD-B1 nachweisen, dass sie die deutsche Sprache kompetent in vielen Situationen des täglichen Lebens und auch im vertrauten beruflichen Umfeld einsetzen können. Dies bedeutet konkret, dass sie fähig sind, alle für sie wichtigen Situationen während eines Aufenthaltes in einem deutschsprachigen Land, aber auch in ihrem eigenen Land, in Situationen, in denen Deutsch erforderlich ist, sprachlich zu meistern. Mit der Prüfung ZD-B1 zeigen die Teilnehmenden auch, dass sie die Sprache Deutsch mit ihren unterschiedlichen regionalen Besonderheiten verstehen. Das soll nicht bedeuten, dass Dialekt abgefragt oder getestet wird, aber es soll bereits auf dieser Stufe ein deutliches Zeichen gesetzt werden, damit sich die Lernenden darauf einstellen können, in Deutschland, in Österreich und in der Schweiz unterschiedliche Aussprachen und teilweise verschiedene Wörter für die gleichen Dinge vorzufinden. 
ZD-B1 beschreibt, was ein Erwachsener können muss, um im persönlichen Alltag, aber auch in der Arbeitswelt problemlos kommunizieren zu können. Daraus ergeben sich Lernziele, die sowohl das Verstehen von gesprochener und geschriebener Sprache als auch das Sprechen und Schreiben umfassen. (Dazu gehören auch Wort- und Themenlisten und ein Katalog von Grammatikphänomenen). Da diese vier Fertigkeiten nur sehr selten allein benötigt werden, müssen sie für das Lernen möglichst in einem $\mathrm{Zu}$ sammenhang dargestellt werden. Zwar ist das Lernen von Grammatik und Wortschatz weiterhin notwendig, doch nur im Zusammenhang von Texten und Szenarien. Von diesen Lernzielen sind die Prüfungsmodelle abgeleitet. Die Prüfungskandidaten sollen dabei weniger bekunden, dass sie Grammatik und Wortschatz gelernt haben, sondern dass sie in verschiedenen Situationen des Lebens sprachlich richtig und verständlich reagieren können, das heißt, dass sie mit anderen Menschen, die die deutsche Sprache sprechen, Beziehungen aufnehmen und sich verständigen können.

\section{SO FUNKTIONIERT DIE PRÜFUNG ZUM ZERTIFIKAT DEUTSCH}

Die Prüfung besteht aus einem schriftlichen Teil, der insgesamt 150 Minuten dauert und die Fertigkeiten: Leseverstehen, Sprachbausteine, Hörverstehen, schriftlicher Ausdruck (Brief) umfasst, und aus einem mündlichen Teil, der 15 Minuten dauert. Die mündliche Prüfung wird - je nach Institution - als Einzelprüfung oder als Paarprüfung durchgeführt. Im ersten Fall unterhält sich der Kandidat mit einem der beiden Prüfer, im zweiten Fall sprechen die beiden Kandidaten miteinander. Die beiden Kandidaten haben 20 Minuten Zeit, um sich auf das Gespräch vorzubereiten. Im Falle der Paarprüfung dürfen die Kandidaten während der Vorbereitungszeit nicht miteinander sprechen.

Jeder Aufgabe sowohl bei dem schriftlichen als auch bei dem mündlichen Teil werden Punkte zugeteilt, die man für jede korrekte Lösung, Antwort oder Aufgaben zum schriftlichen Ausdruck gewinnen kann. Die Punktenzahl für die ganze Prüfung beläuft sich auch 300 , wobei 225 für den schriftlichen Teil und 75 für den mündlichen Teil vorgesehen werden. Um die Prüfung bestanden zu haben, muss der Kandidat mindestens $60 \%$ der Punktenzahl in dem schriftlichen (d.h. 135 Punkte) und auch 60\% in dem mündlichen Teil (d.h. 45 Punkte) erreicht haben. Hat der Prüfungsteilnehmer in beiden Prüfungsteilen die Bestehensgrenze erreicht, werden die Teilergebnisse addiert und daraus die Gesamtnote ermittelt: 270-300 Punkte - sehr gut, 240-269,5 Punkte - gut, 210-239,5 Punkte - befriedigend, 180-209,5 Punkte - ausreichend.

Die Einstufungstests und Lernfortschrittstests haben eine besondere Bedeutung auf die spätere, gezielte ZD-Prüfungsvorbereitung. 


\section{EINSTUFUNGSTESTS}

Im Schulunterricht haben die Lehrer vor allem mit relativ homogenen, schon festgelegten Lerngruppen zu tun. Innerhalb der Gruppe gibt es oft recht große Unterschiede, die von dem Lehrer erkannt und durch eine entsprechende Unterrichtsplanung ausbalanciert sein sollten. Bei dem Schulunterricht, wo die Schüler den Klassen zugeordnet sind, wäre es sinnvoll einen Einstufungstest durchzuführen, so wie es bei der Erwachsenenbildung üblich ist. Dank dem Einstufungstest können die Lerner oder Bewerber auf verschiedene Niveaugruppen verteilt werden. Es ist ratsam für die Kursleiter die Einstufungstests für kleinere Gruppen oder Klassen selbst zu erstellen. Dank den ausgewählten und einheitlichen Fragen mit gleichartigen Mitteln können Informationen über den Kenntnisstand zu Beginn eines Kurses gewonnen werden. Wichtig dabei ist, dass alle Teilnehmer, die gleichzeitig eingestuft werden sollen, denselben Test unter ähnlichen Bedingungen schreiben. Je nach den späteren Lernzielen können jeweils andere Inhalte (z.B. Wortschatz) oder Fertigkeiten im Vordergrund stehen.

\section{LERNFORTSCHRITTSTESTS}

Lernfortschrittstests können immer wieder z.B. nach der Behandlung eines Lehrbuchabschnitts durchgeführt werden. Aus Zeitgründen ist es für die Lehrer, die einige Jahre lang mit demselben Kursbuch arbeiten oder die gleiche Niveaustufe unterrichten ratsam, Lernfortschrittstests zu entwerfen, die dann später mehrmals eingesetzt werden. Die Lernfortschrittstests können so flexibel gestaltet werden, dass sie den besonderen Bedürfnissen der jeweiligen Gruppe Rechnung tragen. So können einzelne Teile dementsprechend z.B. den Texten, Themen, dem Wortschatz oder der Grammatik angepasst werden. Die informellen Lernfortschrittstests können auch als gute Vorbereitung auf die formelle ZD-Prüfung eingesetzt werden. Im Gegensatz zu formellen Prüfungen ist es bei Lernfortschrittstests im Unterricht nicht notwendig und oft aus Zeitgründen fast ausgeschlossen, bei jedem Test Aufgaben zu allen Fertigkeiten anzubieten. Es soll von dem Lehrer im Auge behalten werden, dass im Laufe des Kurses zu allen Lernzielen des Unterrichts Testaufgaben angeboten werden, um wirklich ein kompaktes Leistungsbild der Schüler zu erhalten. So soll zu Beginn eines Kurses ein Plan der Lernfortschrittstests zu einzelnen Fertigkeiten über den zur Verfügung stehenden Zeitraum erarbeitet werden. Aus Zeitgründen können dann einzige Teile der Lernfortschrittstestes in einer Form der ZD-Prüfungsaufgaben angeboten werden. Einerseits wird der Lernfortschritt getestet, andererseits 
dient der Test als gute Vorbereitung auf die Art und Weise einer Aufgabe der ZD-Prüfung und auf die ZD-Prüfung selbst. Es ist dabei den Lehrern bei den ZD-Vorbereitungskursen zu empfehlen, die Aufmerksamkeit der Kursanten entweder vor oder nach dem Test auf die Aufgabenart zu lenken, so dass die KursteilnehmerInnen immer wieder auf die Form der Prüfung hingewiesen werden. Wie bereits erwähnt, werden die Lernfortschrittstests von dem Unterrichtenden selbst entworfen und oft ohne besondere Vorbereitung "ad hoc" durchgeführt. So wird kontinuierlich die Kontrolle des Lernprozesses dokumentiert und die vorhandenen Defizite im weiteren Unterricht nachgeholt. Bei entstandenen Defiziten kann die Perspektive der Testkandidaten durch den Lehrer übernommen werden und die Bearbeitung der Testaufgaben simuliert werden. Auf diese Weise wird jeder Test auf zwei Ebenen durchgearbeitet: in der Simulationsphase erfahren die Lehrer, welche Anforderungen die Tests an die Testpersonen stellen; in der Reflexionsphase reflektieren die Lehrer die Testformen, Textergebnisse und die Bewertung (vgl. Bolton 1996: 5). Die Lernfortschrittstests können bestimmt zur Überwindung der Angst vor der Prüfung und damit zur Erhöhung der Erfolgsaussichten beitragen.

\section{AUFGABENFORMEN DER ZD-PRÜFUNG UND DIE PUNKTENVERGABE}

Bei den Test und Prüfungen werden verschiedene Formen von Aufgaben verwendet. Man unterscheidet drei Formen der Aufgaben nach dem Grad der Offenheit: offene, halboffene und geschlossene Aufgaben.

Bezogen auf die ZD-Prüfung lässt sich feststellen, dass bei dieser Prüfung zwei Formen der Prüfungsaufgaben nach dem Grad der Offenheit angeboten werden und zwar: offene (schriftlicher und mündlicher Ausdruck) und geschlossene (alle Teile des Leseverstehens, des Hörverstehens und der Sprachbasteine). Die meisten ZD-Prüfungsaufgaben sind die geschlossenen Aufgaben. Bei den geschlossenen Aufgaben wird von den Kandidaten verlangt, aus den vorgegebenen Antworten die richtige Antwort auszuwählen. Die Kandidaten brauchen dabei keine Antwort selbständig zu formulieren, sondern die richtige Antwort zu erkennen. Zu den typischen Formen der geschlossenen Aufgaben gehören Multiple-choice-, Ja/Nein- (richtig/falsch) und Zuordnungsaufgaben. Es ist empfehlenswert für die Lehrer bei verschiedenen Lernfortschrittstests diese Form der Aufgaben aus drei Gründen eizusetzen:

1. Diese Aufgabenform ist zeitsparend bei der Bewertung, was bei einer größeren Gruppenzahl für den Lehrer von großer Bedeutung ist. 
2. Der Bewertung der geschlossenen Aufgaben (wenn sie richtig entworfen sind) kann kaum Subjektivität vorgeworfen werden - die Aufgaben werden objektiv bewertet, weil deren Lösungen vorher ein Punktwert festgelegt wird (obwohl es oft vorgeworfen wird, dass diese Aufgabenform an sich nicht objektiv ist, weil die Zufallschance der richtigen Lösung ziemlich hoch ist).

3. Der letzte Grund zum Einsetzen der geschlossenen Aufgaben ist die Häufigkeit des Auftretens dieser Aufgabenform bei vielen Sprachprüfungen (das Beispiel der B1-Prüfung kann eine Bestätigung für diese These sein).

Anbei ein paar Beispiele der geschlossenen Aufgaben aus den meist verwendeten Lehrwerken der Niveaustufe B1:

- Multiple-choice-Aufgaben:

"EM Brückenkurs - B1": KB: 1f,j,l/46; 2 b, c, e, f/94; AB 11/91, "Studio d - B1": 1b/162, 8/181, TEST/200,

„Tangram aktuell 3 - B1" (Lektionen 1-4): A3/2; 10/60; Test S.107

- Ja/Nein-Aufgaben - Richtig/Falsch-Aufgaben:

„EM Brückenkurs - B1“: KB: 3/54; 4/103; 2/22,

"Studio d - B1": 1b/178, TEST/202,

„Tangram aktuell 3 - B1“(Lektionen 1-4): D3/8; 12/61; 9/99;

- Zuordnungsaufgaben:

„EM Brückenkurs - B1": KB:3/83;3/30; AB:3/36,

"Studio d - B1": 1/38, 3/56, 2b/163, 2/154,

„Tangram aktuell 3 - B1" (Lektionen 1-4): E2/11; 11/61; 12/100; 1/111.

Offene Aufgaben eignen sich vor allem zum Überprüfen der produktiven Sprachleistungen und werden als valides Verfahren zum Testen des schriftlichen und mündlichen Ausdrucks eingesetzt. Ein gutes Beispiel für offene Aufgabe zum Testen des schriftlichen Ausdrucks kann eine Bilderbeschreibung sein. Hier bewähren sich die Bildergeschichten aus der Reihe "Vater und Sohn" und ähnliche, die auf verschiedenen Fortschrittsstufen die Schüler zu einer schriftlichen Produktion auffordern. Als eine typische Aufgabe der Art ist das Schreiben eines Briefes nach vorgegebenen Stichpunkten, das immer zu der Aufgabe der ZD-Prüfung gehört. Diese Form der Aufgabe ist bei den Schülern und Lehrern nicht immer beliebt, weil sie von beiden Seiten einen großen Zeitaufwand - sowohl beim Schreiben als auch beim Bewerten - erfordert. Bei offenen Aufgaben geraten leider die beiden Gütekriterien der Bewertung: Validität und Objektivität in Konflikt. Durch die präzise formulierten Bewertungskriterien kann die Subjektivität der Bewertung eingeschränkt werden. Bei der Vorbereitung auf den schriftlichen Ausdruck soll von dem Lehrer auf drei Bewertungskriterien hingewiesen werden: Berücksichtigung der Leitpunkte, kommunikative Gestaltung und 
formale Richtigkeit. Bei dem Kriterium kommunikative Gestaltung werden bewertet: die sinnvolle Anordnung der Leitpunkte, die Verknüpfung der Sätze/ Äußerungseinheiten, die inhalts- und adressenbezogene Ausdrucksweise, der Adressenbezug (Datum, Anrede, Gruß- / Abschiedsformel). Bei dem Kriterium der formalen Richtigkeit werden Syntax, Morphologie und Orthographie bewertet. Alle Kriterien werden mit Buchstaben A, B, C oder D bewertet, den auch Punkte zugeschrieben werden: A - 5 Punkte, B - 3 Punkte, C - 1 Punkt, D - 0 Punkte. Zu der Gesamtpunktzahl können bis zu zwei Zusatzpunkte hinzugefügt werden: es kann jeweils 1 Zusatzpunkt vergeben werden, wenn die Arbeit überdurchschnittlich für B1 ist: in Bezug auf die sprachliche Vielfalt (Wortschatz und Strukturen) und den Umfang (inhaltliche Gestaltung). Die Gesamtpunktzahl (3 Kriterien plus mögliche Zusatzpunkte) wird in der Prüfungszentrale mit 3 multipliziert. Maximale Punktenzahl für den schriftlichen Ausdruck sind 45 Punkte (inklusive Bonuspunkte).

Der mündliche Ausdruck wird auch mit Hilfe der offenen Aufgaben trainiert und dann bei der ZD-Prüfung in allen drei Teilen in dieser Form getestet. Die Prüfer benoten den Teil ähnlich wie den schriftlichen Ausdruck mit den präzise formulierten Bewertungskriterien. So bei dem Einüben allen drei Aufgaben zum mündlichen Ausdruck soll auf folgende vier Hauptbewertungskriterien geachtet werden: Ausdrucksfähigkeit, Aufgabenbewältigung, Aussprache und Intonation.

Alle vier Kriterien werden mit Buchstaben A, B, C oder D bewertet, wobei bei den ersten zwei Kriterien: A - voll angemessen, B - im Großen und Ganzen angemessen, C - kaum noch akzeptabel und D - durchgehend nicht ausreichend, bedeuten. Beim Kriterium formale Richtigkeit macht die bewertete Person: A - keine oder nur sehr vereinzelte Fehler, B - wenige Fehler, die die Verwirklichung der Redeabsicht aber nicht beeinträchtigen, $\mathrm{C}$ - viele Fehler, die die Verwirklichung der Redeabsicht erheblich beeinträchtigen und D - so viele Fehler, dass das Verständnis nicht mehr möglich ist. Bei der Aussprache und Intonation: Abweichungen von Aussprache und Intonation: A - beeinträchtigen das Verständnis nicht, B - erschweren gelegentlich das Verständnis, C - erschweren das Verständnis erheblich und D - machen das Verständnis nahezu unmöglich.

Diese Liste der Kriterien wird als Hilfe bei der Bewertung allen drei Teilen des mündlichen Ausdrucks verwendet. Den oben vorgeführten Buchstaben entsprechen dann die Punkte, die auch später je Aufgabe multipliziert werden. So für A werden 4 Punkte, für B - 3, für C - 1 und D - 0 Punkte vorgesehen (bei Aussprache und Intonation für A und B ein Punkt weniger).

Ein paar Beispiele der offenen Aufgaben aus den meist verwendeten Lehrwerken der Niveaustufe B1: 
„EM Brückenkurs - B1": AB: 10/80; 12/81, "Studio d - B1": 4/48, 5/157, TEST/2003,

"Tangram aktuell 3 - B1" (Lektionen 1-4): 23/66; D3/23; 18/103.

\section{HILFE BEI DER PRÜFUNGSVORBEREITUNG}

In dem neokommunikativen Unterricht kann man nicht von einer vorherrschenden Vorbereitungsmethode hinsichtlich der ZD-Prüfung sprechen. Die Lehrer und auch die Lerner sollen sich darüber im Klaren sein, dass es "keine Wundermethode" für die Prüfungsvorbereitung gibt.

An dieser Stelle sollen zwei Arten von Kursen oder Vorbereitung auf die Prüfung ausgesondert werden: gezielte Vorbereitung auf die Prüfung, wobei nur Probeprüfungssätze gelöst und Prüfungsstrategien entwickelt werden, und die Kurse, die Evaluation des Sprachenfortschritts mit gleichzeitigem Anstreben der Niveaustufe im Auge behalten. Bei der ersten Art der Kurse werden ausschließlich Modelltests von dem zukünftigen Prüfling gelöst; man basiert dabei auf seinem Allgemeinsprachwissen. Dazu gibt es auf dem Markt eine breite Reihe von Publikationen, die die ZD-Prüfung beschreiben und zahlreiche Aufgaben diesbezüglich anbieten: „Mit Erfolg zum Zertifikat Deutsch“, "So geht's zum ZD”, "Tangram ZD“, "Themen neu ZD“. Man betrachtet die Vorbereitung als Selbstarbeit oder den Abschlussteil eines Kurses. Ein Intensivkurs, der in Polen oft als Semester- oder Jahreskurs angeboten wird, wäre sehr monoton, wenn man dabei nur Prüfungssätze lösen würde. Als Alternative für die Evaluation bei Kursen oder Kursteilen, wo nur Prüfungssätze durchgearbeitet werden, wäre eine Form der Ergänzung der Prüfungsaufgaben oder der Stimulierung hinsichtlich des Sprachengebrauchs. Und so kann man den Aufgaben des schriftlichen Prüfungsteils wie LV1, LV2, HV1 und HV2 neue Aufgabenformen hinzufügen und so z.B. die KursteilnehmerInnen auffordern, ihre Meinung zum Aufgabenthema zu äußern. Angebracht dabei wäre Paararbeit, Gruppenarbeit und schließlich Plenumsdiskussion über die Schlussfolgerungen oder Ideen. So haben die KursteilnehmerInnen, die sich auf die B1-Prüfung vorbereiten, eine Möglichkeit der Selbstevaluation und des realen und nicht nur gesteuerten Sprachengebrauchs. Gleichzeitig bereiten all die mündlichen Kommentare, Diskussionen, Aussagen, Stellungnahmen usw. auf den mündlichen Teil der B1-Prüfug vor. Bei der monotonem Lösen der Richtig/Falsch-Aufgaben, d.h. Ankreuzen im Alternativverfahren, haben die Kursanten immer wieder nur mit geschlossenen Aufgaben zu tun. Die meisten von diesen Aufgaben haben einen rezeptiven Charakter. An dieser Stelle wird dann von dem Kursleiter verlangt, von den geschlossenen, rezeptiven Aufgaben zu interaktiven 
und letztendlich produktiven Aufgaben, wo die Sprache richtig produziert wird, überzugehen.

Bei weiteren Kursen, die oft als Zertifikatskurse oder Kurse, die als Vorbereitung auf die Prüfung genannt werden und bei denen das leitende Kursbuch eins aus der Reihe B1-Kursbücher ist, haben die Kursleiter eine umgekehrte Aufgabe als die oben beschriebene. Diesmal wird von dem Kursleiter erwartet, an verschiedenen Stellen, wo man eine auf die Prüfung vorbereitende, "passende" Übung einsetzt, die einzusetzen. Am Kursanfang wäre den Lehrern empfohlen, die Prüfungsaufgaben einzusetzen ohne die KursteilnehmerInnen auf die Art der Übung hinzuweisen. So wird die Aufgabe von den KursteilnehmerInnen spontan gelöst. Mit der Zeit sollen sich die Kursteilnehmer darüber im Klaren sein, wann sie mit einer Prüfungsaufgabe zu tun haben. Solche Verfahrensweise weckt bei den KursteilnehmerInnen Zusatzmotivation, Konzentration und Aufmerksamkeit.

Den KursteilnehmerInnen sollen alle Arten der Aufgaben angeboten werden, auch solche, die nicht den Aufgabenformen der exakten Prüfung entsprechen. Dank der Aufgabenvielfalt findet das komplexe Beherrschen der Fremdsprache statt - mit jeder Form des Weiterlernens werden'neue Fähigkeiten und Fertigkeiten hinzugewonnen.

Die Schüler oder Prüflinge sollen auch in der Lage sein, während des Tests oder der Prüfung die Arbeitsanweisungen zu den Testaufgaben problemlos zu verstehen. Leider werden die Arbeitsanweisungen oft zu kompliziert oder missverständlich formuliert und dadurch die Aufgaben falsch gelöst. Es ist von großer Bedeutung, die Schüler schon im Anfängerunterricht mit der formellen Arbeitsanweisungssprache vertraut zu machen und die Arbeitsanweisungssprache immer wieder in verschiedener Form bei jedem Test oder Lernfortschrittskontrolle einzusetzen.

Um den Prüfungsstress abzubauen sollen die Lernenden in der Vorbereitungsphase mit verschieden Formen der Aufgaben konfrontiert werden. Die im Schulraum von den Lernenden gemachten Tests sind oft mit Situationsstress verbunden. Dank den im Internet angebotenen E-Tests können sich die Lernenden stressfreier mit den Testaufgaben beschäftigen. Auch das Angebot von den CD-ROMs erleichtert und stimuliert die Prüfungsvorbereitung. Unabhängig davon, wo und wie die Lernenden das Wissen vertiefen, soll im Vordergrund immer das angestrebte Ziel stehen.

\section{LITERATURVERZEICHNIS}

Bausch, K.-R./Königs, F.G., 1985. (Er)Werben und (Er)Lernen. Eine Antwort auf zwei Antworten. In: Die neueren Sprachen, 84, 218-233. 
Bausch, K.-R./Krumm, H.-J. (Hrsg.), 1995. „Sprachlehrforschung”. In: Bausch, K.-R./Christ, H. /Krumm, H.-J. (Hrsg.), Handbuch Fremdsprachenunterricht. Tübingen und Basel: Francke Verlag: 7-12.

Bolton, S., 1996. Probleme der Leistungsmessung. Lernfortschrittstests in der Grundstufe. Fernstudieneinheit 10. Berlin u.a.: Langenscheidt.

Chudak, S., 2007. Lernerautonomie fördernde Inhalte in ausgewählten Lehrwerken DaF für Erwachsene. Frankfurt am Main: Peter Lang.

Ciepielewska, L., 2002. „Die Bedeutung der Lehrwerkanalyse für den modernen Fremdsprachenunterricht". In: Glottodidactica. Volume XXVII. Poznań: Wydawnictwo Naukowe UAM, 65-67.

Council of Europe, 1997. European language portfolio: proposals for development. Strasbourg: Council of Europe.

Dietrich, I./Gäßler, R./Schilder, H., 1983. Der Fremdsprachenunterricht auf dem Wege der Schülerorientierung. In: Die Neueren Sprachen 82/3. (1983), 227-239.

Dłużniewski, S., 1992. Testy i zadania egzaminacyjne z jezyka niemieckiego. Warszawa: PWN.

Doyé, P., 1988. Typologie der Testaufgaben für den Unterricht Deutsch als Fremdsprache. Berlin u.a.: Langenscheidt.

Doyẻ, P., 1995. „Funktionen und Formen der Leistungsmessung”. In: Bausch, K.-R./Christ, H. /Krumm, H.-J. (Hrsg.), Handbuch Fremdsprachenunterricht. Tübingen und Basel: Francke Verlag: 277-282.

Europarat. Rat für kulturelle Zusammenarbeit (Hrsg.), 2001. Gemeinsamer europäischer Referenzrahmen für Sprachen: lernen, lehren, beurteilen. Berlin: Goethe-Institut Inter Nationes u.a. Hrsg.).

Kast, B., 1996. Fertigkeit Schreiben. Fernstudieneinheit 12. Berlin/München: Langenscheidt.

Komorowska, H., 1974. Testy w nauczaniu języków obcych. Warszawa: Wydawnictwo Szkolne i Pedagogiczne.

Komorowska, H., 2007. Sprawdzanie umiejętności w nauce języka obcego. Kontrola - Ocena - Testowanie. Warszawa: Fraszka Edukacyjna.

Lienert, G.A., Raatz, U., 1994. Testaufbau und Testanalyse. Weinheim: Beltz, Psychologie Verlags Union.

Macht, K., 1995. Leistungsmessung und Curriculum. In: Bausch, K.-R./Christ, H./Krumm, H.-J. (Hrsg.), Handbuch Fremdsprachenunterricht. Tübingen und Basel: Francke Verlag, 282-285.

Neuner, G., 1995. Die Erstellung von informellen Leistungstests („Schulaufgaben”) mit Hilfe der Übungstypologie. In: Neuner, G./Krüger, M./Grewer, U. (Hrsg.), Übungstypologie zum kommunikativen Deutschunterricht. Berlin u.a.: Langenscheidt.

Sändig, U., 2002. „Aufgabentypen und Testverfahren zum Überprüfen der kommunikativen Kompetenz im Unterricht Deutsch als Fremdsprache". In: Krück, B.I Loeser, K. (2002) Innovationen im Fremdsprachenunterricht 2 Fremdsprachen als Arbeitssprachen. Frankfurt a.M. u.a.: Peter Lang, 175-195.

Schmidt, R., 1994. Tests und Prüfungen. In: Heinrichi, G./Riemer, C. (Hrsg.) (1994): Einführung in die Didaktik des Unterrichts Deutsch als Fremdsprache mit Videobeispielen. Band 2. Schneider Verlag Hohengehren GmbH, 353-395.

Skowronek, B., 1997. Methodologische Rekonstruktion glottodidaktischer

Theorien. Poznań: Wydawnictwo Naukowe UAM.

Vollmer, H.J., 1995. Leistungsmessung: Überblick. In: Bausch, K.-R./Christ, H./Krumm, H.-J. (Hrsg.), Handbuch Fremdsprachenunterricht". Tübingen und Basel: Francke Verlag, 273-277.

Vollmer, H.J., 2003. Leistungsmessung, Lernkontrolle und Selbstkontrolle. In: Bausch, K-R./ Christ, H./Krumm, H.-J. (Hrsg.), Handbuch Fremdsprachenunterricht. Tübingen und Basel: Francke Verlag, 365-370. 